\title{
Factores de riesgo que predisponen a trastornos de la marcha en personas adultas mayores institucionalizadas de 65 a 80 años
}

Risk factors for gait disorders in institutionalized older people from 65 to 80 years old

\begin{abstract}
Karla Vindas Ramírez 1 $\square$; Óscar Araya Quesada 2; Rodrigo Cambronero Román ${ }^{3}$;
Marlen Roselló Araya ${ }^{4}$; Erica Cyrus Barker ${ }^{5}$.

Filiación:

1 Trabajadora independiente San

Pablo Heredia, Costa Rica.

2 Trabajador Independiente. San

Ramón de Alajuela, Costa Rica.

3 Hogar de Ancianos Fray Casiano

de Madrid de Miramar, Puntarenas,

Costa Rica.

4 Instituto Costarricense de Investigación y Enseñanza en Nutrición y Salud- INCIENSA, Cartago, Costa Rica.

5 Universidad Santa Paula, Escuela de Terapia Física, Curridabat, Costa Rica.

Correspondencia: $\square$ Karla Vindas Ramírez, correo electrónico: vindas04@gmail.com

- - - - - - - - - - - -

Abreviaturas: $A D M=$ Alteraciones

de la Marcha; $A M=$ Adulto mayor; $D F=$ Deterioro Funcional; PAM= Persona Adulta Mayor; PAMl= Persona Adulta Mayor Institucionalizada;

$T M=$ Trastornos de la Marcha; VDM= Velocidad de Marcha.

Forma de citar: Vindas Ramírez $K$, Araya Quesada O, Cambronero Román R, Roselló Araya M, Cyrus Baker E. Factores de riesgo que predisponen a trastornos de la marcha en personas adultas mayores institucionalizadas de 65 a 80 años. Rev Ter. 2018;12(2):7-22.

\section{Resumen}

La población envejece a nivel global y considerando que la institucionalización juega un papel muy importante en esta etapa es fundamental considerar las repercusiones que ésta trae consigo en la marcha. El objetivo del estudio fue describir los principales factores que predisponen alteraciones de la marcha (ADM) en personas adultas mayores institucionalizadas (PAMI), con edades entre 65 y 80 años.

Materiales y métodos: estudio de revisión narrativa de la evidencia existente acerca de los factores de riesgo para trastornos de la marcha (TM) en PAMI. Utilizando las bases de datos Cochrane database, MEDES, Scielo, Trip database, Google académico y JAMDA se seleccionaron 40 artículos científicos, publicados entre el año 2013 al 2018, en idioma español e inglés.

Resultados: Según la literatura las ADM en el adulto mayor (AM) más frecuentes son, disminución de la velocidad y longitud de paso, asimetría en el movimiento y seudoclaudicación. Entre los principales factores predisponentes están: el perfil de la persona adulta mayor (PAM), caracterizado por deterioro funcional (DF), discapacidad, dependencia, comorbilidad y edad muy avanzada, el paradigma de atención reactivocurativo de los sistemas de institucionalización que limita el desempeño independiente del usuario, no se enfoca en la prevención y somete al individuo a reglas que obligan al sedentarismo; por último, la infraestructura suele ser deficiente e inadecuada según las necesidades de los residentes.
\end{abstract}

Financiamiento:

Autofinanciado

Conflictos de Interés:

El autor declara no tener conflictos de interés en la presente publicación
Conclusiones: las ADM en AM institucionalizados están influenciadas por factores intrínsecos del usuario, el modelo de atención de los centros de institucionalización y características en infraestructura. 
Palabras claves: adulto mayor institucionalizado, marcha, marcha normal, adulto mayor.

\section{Abstract}

The population ages globally and considering that institutionalization plays a very important role in this stage it is important to consider the repercussions that this brings with it in the march. The objective of the study was to describe the main factors that predispose gait alterations in institutionalized older adults, aged between 65 and 80 years.

Materials and methods: study of the narrative review of existing evidence about risk factors for gait disorders in institutionalized older adults. Using the databases Cochrane database, MEDES, Scielo, Trip database, academic Google and JAMDA, 40 scientific articles were selected, published between 2013 and 2018, in Spanish and English.

Results: according to the literature, the most frequent alterations in gait in the elderly are: decrease in speed and step length, asymmetry in movement and pseudo-claudication. Among the main predisposing factors are: the profile of the elderly person, characterized by functional impairment, disability, dependence, comorbidity and very old age, the paradigm of reactive-curative care of institutionalization systems that limits the user's independent performance, it does not focus on prevention and subjects the individual to rules that force a sedentary lifestyle; finally, the infrastructure is often deficient and inadequate according to the needs of the residents.

Conclusions: the gait disorders in institutionalized older people are influenced by intrinsic factors of the user, the care model of institutionalization centers and characteristics in infrastructure.

Key words: aging, institutionalized, elderly, gait, normal gait.

\section{Introducción}

En el ámbito mundial, el aumento de la esperanza de vida genera la formación de estrategias que permitan cubrir las demandas de las PAM en sus diferentes esferas, tanto física, social, psicológica y funcionalmente ${ }^{1}$. Esta capacidad de vivir más años generalmente se relaciona y desarrolla en conjunto con diferentes procesos degenerativos propios del envejecimiento además de la interacción de los factores ambientales en el desempeño cotidiano de los individuos ${ }^{2}$. Existen diferentes modelos para hacerle frente al cuido y manejo de las personas de la tercera edad con el fin de brindarles un envejecimiento digno e integral. Ante la posibilidad de que el cuido en el entorno inmediato sea imposible surge la institucionalización como una alternativa para satisfacer las necesidades básicas de los usuarios y proporcionar la mayor calidad de vida al sujeto. En Costa Rica la población adulta mayor para el 2017 correspondía a 389840 personas, lo que según el Instituto Nacional de Estadísticas y Censos (INEC) representa el 7,9\% de los habitantes del país ${ }^{3}$, respecto a los adultos mayores institucionalizados tanto en el sector público como privado la cifra asciende a 3379 personas ${ }^{4}$, lo que posiciona a la institucionalización como una opción de cuido de gran importancia.

El ambiente en el que se desarrolle un AM sea en la comunidad o bien un sistema de institucionalización podría proteger o predisponer a alteraciones en 
sus patrones motores. Existe controversia acerca de las diferentes repercusiones cognitivas $y$ funcionales con las que se asocia la estancia en los centros de cuido, principalmente tomando en cuenta las actividades de la vida diaria y la capacidad autónoma de poder desplazarse, específicamente la marcha. Este artículo busca describir los principales factores de riesgo que predisponen ADM de PAMI con edades entre 65 a 80 años, comprendiendo la ejecución de la marcha como un determinante en la condición de salud del paciente geriátrico.

\section{Metodología}

El presente es un estudio narrativo de revisión bibliográfica exhaustiva de la evidencia existente acerca de los factores de riesgo para TM en PAMI con edades entre 65 y 80 años. Utilizando las bases de datos Cochrane database, MEDES, Scielo, Trip database, Google académico y JAMDA se realizó una búsqueda de artículos en inglés y español entre el 2013 y 2018. Se utilizaron las palabras clave "adulto mayor institucionalizado", "marcha", "adulto mayor" (aging, institucionalized, elderly, gait ).

De los artículos incluidos $40 \%$ son estudios descriptivos, transversales o logitudinales; $22,5 \%$ corresponden a revisiones bibliográficas, $20 \%$ representan estudios de cohorte, 10\% pertenece a revisiones sistemáticas - meta-análisis, 5\% incorporan opiniones de expertos y $2,5 \%$ atañe a un ensayo clínico de los temas relacionados. En el cuadro 1 se observa los tipos de estudios incluidos así como la cantidad de artículos y porcentaje correspondiente a cada uno. De la revisión bibliográfica se obtuvo 89 artículos de los cuales se excluyeron 49 por ser estudios de nivel de evidencia muy bajo y con deficiencias metodológicas. De los artículos científicos seleccionados se incluyeron 40 clasificados según el nivel de evidencia de Sackett como sigue: 6 artículos de nivel 1 (15\%), 6 de nivel 2 (15\%), 4 de nivel $3(10 \%), 22$ de nivel $4(55 \%)$ y 2 de nivel $5(5 \%)$.

Cuadro 1: Tipos de estudios incluidos, número de artículos y porcentaje correspondiente a cada uno

\begin{tabular}{|l|c|c|}
\hline \multicolumn{1}{|c|}{ Tipo de estudio } & Artículos & $\%$ \\
\hline $\begin{array}{l}\text { Estudios descriptivos, trans- } \\
\text { versales y longitudinales }\end{array}$ & 16 & $40 \%$ \\
\hline Revisiones bibliográficas & 9 & $22,5 \%$ \\
\hline Estudios de cohorte & 8 & $20 \%$ \\
\hline $\begin{array}{l}\text { Revisiones } \\
\text { cas-meta-análisis }\end{array}$ & 4 & $10 \%$ \\
\hline Opinión de expertos & 2 & $5 \%$ \\
\hline Ensayo clínico & 40 & $100 \%$ \\
\hline Total & $4,5 \%$ \\
\hline
\end{tabular}

Fuente: elaboración propia, 2018.

\section{Resultados}

La deambulación representa en el AM un parámetro de funcionalidad, calidad de vida, independencia, así como la forma de cuantificar su estado de salud. La capacidad de marcha es la habilidad de locomoción del individuo definida a través de progreso o avance, control postural y adaptabilidad, se describe con base a parámetros espaciales y temporales de su ciclo ${ }^{5}$.

Las ADM se definen por una disminución de la velocidad de marcha (VDM), alteración en las características del paso (base, longitud, rangos de movimiento) inestabilidad o modificación en 
la sincronía de ambas extremidades inferiores, generando ineficacia para el desplazamiento y alterando las actividades de la vida diaria6. En la figura 1 se observan los cambios biomecánicos a los que se expone un AM, que afectan el proceso de marcha normal y que podrían representar alteración.

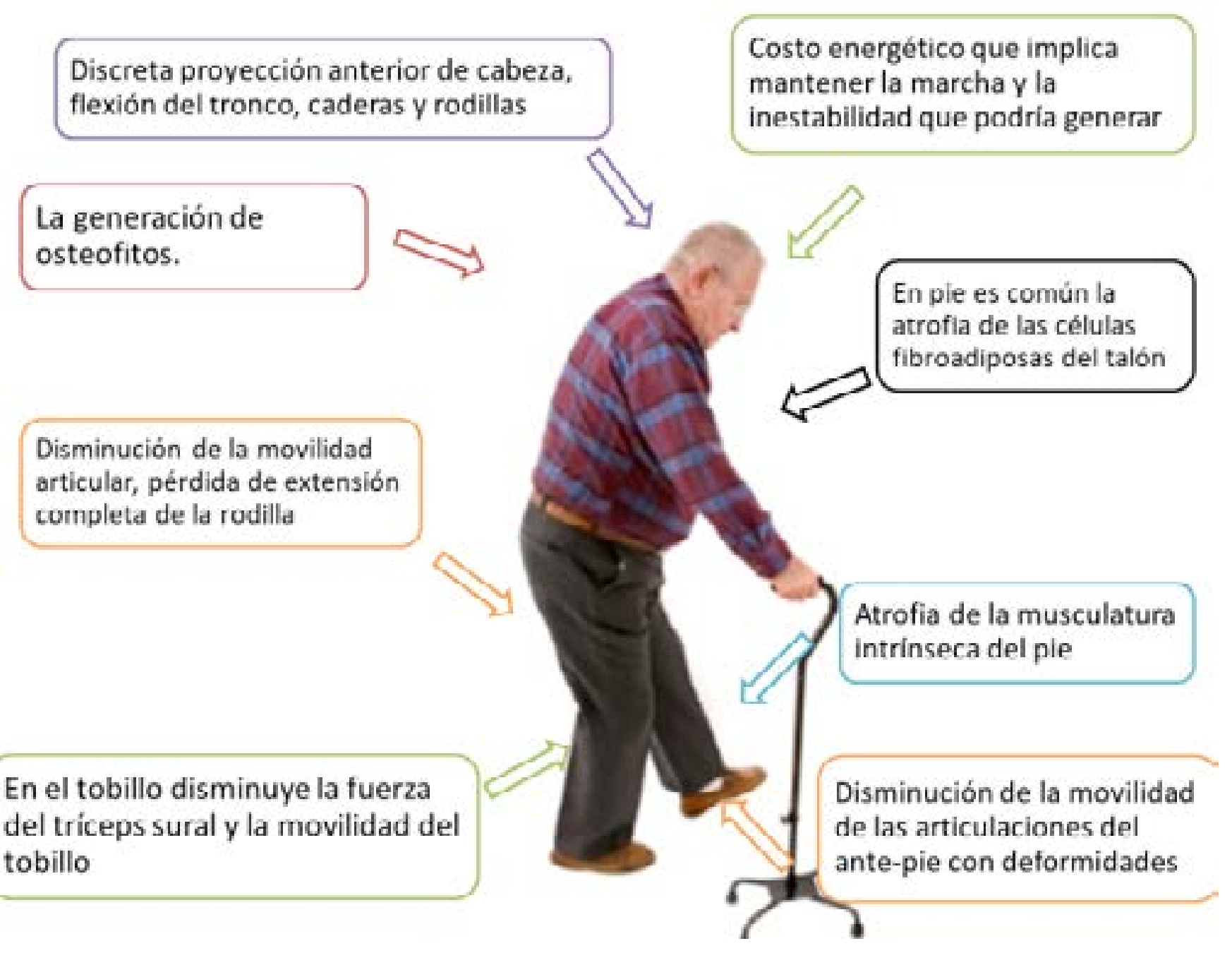

Figura 1: Cambios biomecánicos en la persona adulta mayor que afectan el proceso de marcha.

Fuente: Cerda, 2014. Guerrero M y cols. 2015. Saüch G. 2013.

Según Agudelo y cols.7, entre los 60 y 70 años inicia la manifestación de los cambios normales del envejecimiento en el proceso de marcha, como disminución del balanceo, alteraciones posturales, diminución en la velocidad, cadencia y longitud de paso. En la figura 2 se representa el porcentaje de
PAM con ADM según edad, se puede observar que en la década de los 60 los adultos mayores con TM corresponde a $15 \%$, mientras que a los setentas el porcentaje aumenta a $35 \%$ y a partir de los 85 años $50 \%$ de las PAM presentan algún trastorno para la deambulación ${ }^{6,8-10}$. 


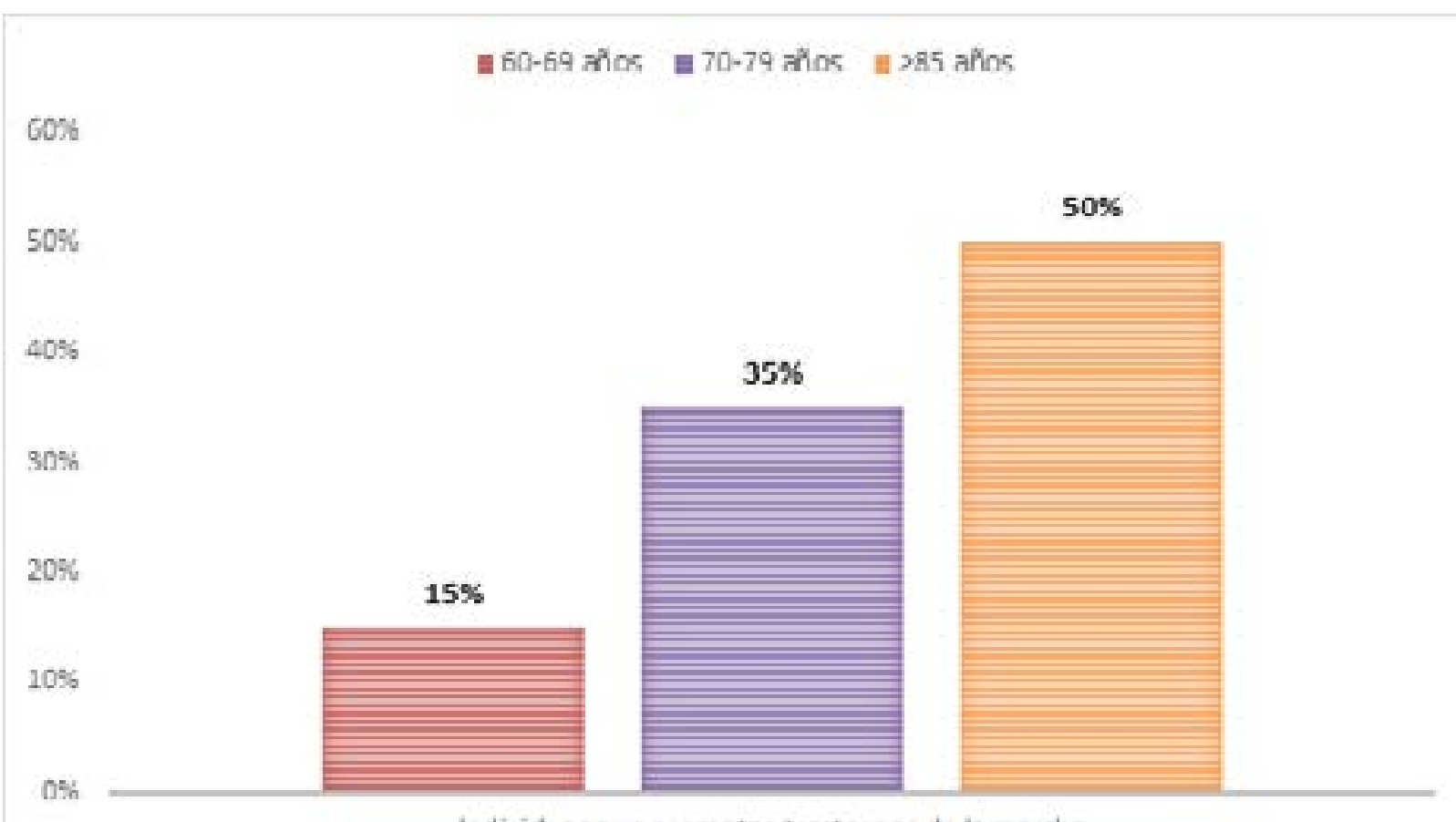

Individuos que presentan tr astornos de la morcho

\section{Figura 2: Porcentaje de personas adultas mayores con alteraciones de la marcha según edad.}

Fuente: Elaboración propia basada en referencias: 6, 8-10.

La VDM menor a $1 \mathrm{~m} / \mathrm{seg}$ es indicador de eventos adversos en la PAM aparentemente sano y si la VDM disminuye hasta menos de $0,8 \mathrm{~m} / \mathrm{seg}$, se puede perder la capacidad de marcha. A partir de los 60 años la velocidad disminuye $1 \%$ por año ${ }^{6}$, principalmente por la sustitución en el número de fibras de contracción rápida de fuerza impulsiva que se ven opacadas por la necesidad del adulto a lograr una mayor estabilidad en la locomoción, cambios que se manifiestan con menor fuerza propulsiva y menor resistencia aeróbica $6,7,11$.

El promedio de VDM en PAMI con capacidad para deambular está establecido en $0,48 \mathrm{~m} / \mathrm{s}$ a un ritmo de paso habitual, y de $0,67 \mathrm{~m} / \mathrm{s}$ a una capacidad de velocidad máxima; así pues, se determina la velocidad de $0,2 \mathrm{~m} / \mathrm{s}$ como indicador de extrema fragilidad para PAM no institucionalizadas y de $0,15 \mathrm{~m} / \mathrm{s}$ para aquellas institucionalizadas ${ }^{7}$. A nivel propio del análisis de la marcha, las alteraciones se pueden mostrar en 4 campos: cinemáticos, espaciales, temporales y espaciotemporales.

Algunos TM en PAM según Cerda $^{6}$ son: dificultad en el inicio de la marcha, pérdida de simetría del movimiento entre los dos hemicuerpos, marcha con aumento de la base de sustentación, deambulación con seudoclaudicación (es decir, dolor, debilidad muscular y parestesias de extremidades inferiores al caminar), enlentecimiento de la marcha y marcha con desviación en la trayectoria.

La detección oportuna de ADM permite prevenir el rápido deterioro de la salud delAM con intervenciones que logran una reducción del riesgo de muerte en un $18 \%{ }^{12,13 .}$ El análisis de las ADM con un instrumento objetivo para su diagnóstico es fundamental para una adecuada intervención ${ }^{6}$. 
Factores de riesgo para alteraciones de la marcha

Muchos factores relacionados con la edad tienen efecto en un adecuado patrón de marcha de los AM, la funcionalidad física y cognitiva de la PAMI juega un papel fundamental para la vivencia de una vejez digna y con buena calidad de vida.

El deterioro cognitivo y funcional de los AM al someterse al sistema de institucionalización está influenciado por factores intrínsecos así como por características propias de la institución ${ }^{14-20}$.

Dentro de los factores intrínsecos varios autores aseguran que la tipología del AM institucionalizado comprende características como: DF- dependencia - discapacidad, edad muy avanzada, género femenino, comorbilidad, deterioro cognitivo de moderado a severo, alto riesgo de caídas, depresión, fragilidad, incontinencia, polifarmacia, riesgo social (viudez), inactividad-sedentarismo, dolor, aislamiento y sarcopenia ${ }^{8,14-18,20-32}$. En el cudro 2 se detalla el perfil de las PAMI de acuerdo a lo descrito por varios autores.
De igual manera existen otros factores que involucran a la PAMI e influyen en el desempeño de la marcha, como el que los residentes poseen más limitaciones en ejecutar un plan de ejercicios que AM no institucionalizados ${ }^{11,22,28,32-37}$ están expuestos a una deprivación sensorial $14,15,18,29,30,38$ y que a su vez generan procesos de adaptación sociales y psicológicos más lentos y con una disminuida capacidad de resolución $7,16,18,20,39,40$.

Los factores relacionados con los sistemas de institucionalización comprenden algunas características de estos sistemas que se han identificado como coadyuvantes en el DF y cognitivo de PAMI ${ }^{15-19}$. Entre ellos se menciona más que todo el paradigma de atención que se utiliza en la mayoría de las residencias de larga estancia que es reactivo-curativo, el cual no se enfoca en la prevención de alteraciones por el contrario prevalecen los pensamientos de sobre protección y estereotipos, provocando un exceso de control sobre los usuarios $14-16,18,22,23,27,28,30,32,34,38,39,41,42$.

\section{Cuadro 2: Perfil de las personas adultas mayores institucionalizadas}

\begin{tabular}{|c|c|c|}
\hline Características & Definición & Observaciones \\
\hline 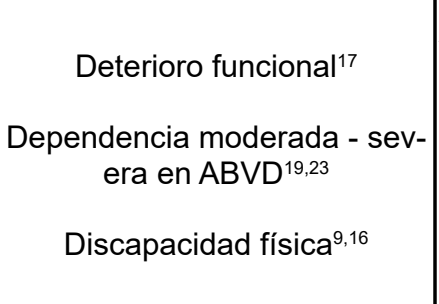 & $\begin{array}{c}\text { Aumento de la dependencia en la real- } \\
\text { ización de las ABVD } \\
\text { Dependencia para la realización de } 3 \\
\text { o más ABVD }{ }^{18} \text {. } \\
\text { Limitación para una movilidad inde- } \\
\text { pendiente }\end{array}$ & $\begin{array}{l}\text { El } 40 \% \text { de los residentes sufren deterioro de las ABVD } \\
\text { después de un año de estar institucionaliza- dos. } \\
\text { Existe mayor deterioro después de un año de ingresar al cen- } \\
\text { tro }(p<.0001)^{19}\end{array}$ \\
\hline Edad muy avanzada $8,14,17,18,21,37$ & $\begin{array}{l}\text { Los estudios consultados coinciden en } \\
\text { que la edad de los usuarios de insti- } \\
\text { tuciones es mayor a los } 80 \text { años. }\end{array}$ & $\begin{array}{l}\text { En Francia el promedio de edad de las personas institucional- } \\
\text { izadas es de } 85 \text { años }(80,01+/-7,35, p=0,73)^{19}\end{array}$ \\
\hline Género femenino $8,14,21,37$ & $\begin{array}{l}\text { Los estudios consultados coinciden } \\
\text { en que la prevalencia de la población } \\
\text { femenina en los centros de institucio- } \\
\text { nalización es mayor que la masculina, } \\
\text { en un } 64,9 \% p=0,2721 \text {. }\end{array}$ & La población masculina corresponde a $35,1 \%{ }^{21}$. \\
\hline Comorbilidad $^{14,21,37}$ & $\begin{array}{c}\text { Presencia de } 5 \text { o más enfermedades } \\
\text { distintas en un adulto mayor, el } 55 \% \\
\text { de la población residente presenta } \\
\text { comorbilidad }{ }^{21} \text {. }\end{array}$ & $\begin{array}{l}\text { Mayor prevalencia se encuentran: demencia23, diabetes }{ }^{23} \text {, en- } \\
\text { fermedades cardiovasculares, enfermedad pulmonar obstructi- } \\
\text { va crónica, cáncer, secuelas de evento cerebro vascular16. }\end{array}$ \\
\hline
\end{tabular}




\begin{tabular}{|c|c|c|}
\hline Características & Definición & Observaciones \\
\hline $\begin{array}{l}\text { Deterioro cognitivo de mod- } \\
\quad \text { erado a severo }{ }^{14,17-19,23}\end{array}$ & $\begin{array}{l}\text { Disminución de las funciones cogni- } \\
\text { tivas como parte de un proceso neu- } \\
\text { rodegenerativo del envejecimiento }{ }^{33} \text {. } \\
\text { El deterioro cognitivo es un predictor } \\
\text { importante de institucionalización }{ }^{18} \text {. }\end{array}$ & $\begin{array}{c}\text { El desempeño en el Mini Mental State Exam de institucional- } \\
\text { izados tiende a ser peor que el de la población en comunidad } \\
\text { disminuye } 2,8 \text { puntos }(p<.0001) \text { de la evaluación inicial a la } \\
\text { segunda evaluación }{ }^{18 .}\end{array}$ \\
\hline Alto riesgo caída $8,23,37$ & $\begin{array}{c}\text { Las caídas afectan de un } 30 \text { a } 50 \% \\
\text { de la población institucionalizada, } \\
\text { con un promedio de } 1,5 \text { caídas por } \\
\text { año }{ }^{8} \text {. }\end{array}$ & $\begin{array}{l}\text { Existe relación entre las caídas y los resultados en el test } \\
\text { Tinetti }(\mathrm{OR}=0,93 ; 95 \% \text { IC: } 0,87-0,98)^{8} \text {. }\end{array}$ \\
\hline Depresión ${ }^{14,18,19}$ & $\begin{array}{l}\text { Existe una relación directa entre la } \\
\text { capacidad física-funcional, incidencia } \\
\text { de caídas y la depresión. }\end{array}$ & $\begin{array}{l}\text { Del } 30-40 \% \text { de resientes son afectados por síntomas depre- } \\
\text { sivos (18). OR } 1,45 \text {, IC } 1,16-1,81, p=0,005^{16 .}\end{array}$ \\
\hline Fragilidad 8,37 & $\begin{array}{c}\text { Estado aumentado de vulnerabilidad } \\
\text { que incrementa el riesgo a sufrir } \\
\text { eventos adversos } \\
\text {. }\end{array}$ & $\begin{array}{l}\text { Los resultados adversos en las pruebas para definir fragilidad } \\
\text { predicen el riesgo de muerte en un año en los residentes } \\
\qquad O R=1.94 ; \text { IC: } 95 \% 1,21-3,09^{8}\end{array}$ \\
\hline Incontinencia $15,17,19,23$ & $\begin{array}{l}\text { La prevalencia de incontinen- } \\
\text { cia urinaria es mayor en un } 43 \% \\
\qquad(p=<.001)^{15}\end{array}$ & $\begin{array}{l}\text { La incontinencia fecal es prevalente en un } 23 \% \text { de la pobla- } \\
\text { ción }^{15} \text {. }\end{array}$ \\
\hline Inactividad, sedentarismo ${ }^{18,34}$ & $\begin{array}{l}\text { Los residentes suelen pasar el } 75 \% \\
\text { del día en actividades sedentarias }{ }^{22} \text {. }\end{array}$ & $\begin{array}{l}\text { Existe una mejora en la capacidad física del individuo si el } \\
\text { mismo aumenta el nivel de actividad física }{ }^{34}\end{array}$ \\
\hline Polifarmacia ${ }^{8,16}$ & $\begin{array}{c}\text { Utilización de } 5 \text { medicamentos o más } \\
\text { por una persona o bien la utilización } \\
\text { de medicamentos en un usuario de } \\
\text { manera innecesaria. OR } 1,30, \text { IC } \\
1,06-1,60, p=0,016^{16} .\end{array}$ & $\begin{array}{l}\text { Fármacos más utilizados: psicotrópicos (OR 1,30, Cl 1,06- } \\
\qquad 1,60, p=0,016) \text { y antidepresivos }{ }^{16}\end{array}$ \\
\hline $\begin{array}{l}\text { Viudez, soltería, separación, } \\
\text { divorcio }^{14,21}\end{array}$ & $\begin{array}{l}\text { El vivir solo representa vulnerabilidad } \\
\text { social y familiar }{ }^{14} \text {. }\end{array}$ & El $59 \%$ presenta alguna de estas condiciónes $p=<.001^{21}$. \\
\hline Aislamiento $^{14}$ & $\begin{array}{l}\text { Interacción social y familiar muy es- } \\
\text { casa }^{14} \text {. }\end{array}$ & $\begin{array}{l}\text { Hay mejor calidad de vida cuando se tiene mayor apoyo } \\
\text { emocional y social }(p=.004)^{14}\end{array}$ \\
\hline Sarcopenia ${ }^{8,33}$ & $\begin{array}{c}\text { Disminución de la masa muscular } \\
\text { que genera pérdida de fuerza mus- } \\
\text { cular y aumenta el deterioro funcio- } \\
\text { nal }{ }^{33} \text {. }\end{array}$ & $\begin{array}{l}\text { Presentar sarcopenia aumenta el riesgo de mortalidad. (OR= } \\
\qquad 1,7, \text { IC } 95 \%: 1,1-2,92 ; p=0,03)^{8}\end{array}$ \\
\hline Dolor $^{20}$ & $\begin{array}{l}\text { El } 53 \% \text { de la población institucionali- } \\
\text { zada indica sentir dolor }{ }^{20} \text {. }\end{array}$ & $\begin{array}{c}\text { Influye directamente en la autopercepción de calidad de vida } \\
\text { del usuario }\end{array}$ \\
\hline
\end{tabular}

$A B V D$ : actividades básicas de la vida diaria

Fuente: elaboración propia basada en referencias 8, 14-18, 20-29, 31, 32, 34. 
En relación con lo anterior, en la institucionalización se establecen reglas que provocan que los usuarios permanezcan la mayor parte del tiempo en comportamientos sedentarios, ya que se les exige mantener descansos por largos periodos y no se motiva a realizar actividades que eleven el gasto metabólico, asimismo se utilizan restricciones físicas y farmacológicas que aumentan la dependencia e inactividad $5-7,9,16,18,31,32,36,38,39,43,44$.

Dentro de este mismo paradigma los horarios de las actividades rutinarias son rígidos, no son sometidos a variaciones para la realización de las actividades básicas de la vida diaria (ABVD) según las necesidades o preferencias de los usuarios, esto propiciado por la sobrepoblación institucionalizada, a la que se debe satisfacer en todas sus necesidades en muy poco tiempo $19,27,30,39,45$.

Otro aspecto propio del sistema es la falta de programas de estimulación tanto física como cognitiva, incluyendo deporte o recreación sumándose a las acciones de los centros de larga estancia que no favorecen a que los usuarios tengan una adecuada capacidad funcional. Aunado a esto la falta de capacitación o especialización del personal de atención directa, los lleva a desconocer las preferencias de los residentes dejando de lado la filosofía de atención centrada en la persona con la que se promueve la autonomía del individuo $9,11,14-16,18,21,22,24,25,27,28,32-34,36-41,43-47$.

En el cuadro 3 se describen las características de los sistemas de institucionalización que predisponen a AM residentes a TM entre los que sobresale el tipo de intervención que se ofrece en las instituciones dado que no es individualizado frente a una población heterogénea y con recursos económicos usualmente limitados ${ }^{15-19,21,23,24,26,32,38,39,44,45}$.

\section{Cuadro 3: Características de los sistemas de institucionalización que predisponen a adultos mayores residentes a trastornos de la marcha}

\begin{tabular}{|c|c|c|}
\hline Aspecto & Características & Observaciones \\
\hline $\begin{array}{l}\text { Paradigma de atención reacti- } \\
\text { vo-curativo }{ }^{17}\end{array}$ & $\begin{array}{l}\text { No se enfoca en la prevención. Pensamientos } \\
\text { de sobre protección y estereotipos. El adulto } \\
\text { mayor es un receptor pasivo de los servicios } \\
\text { al satisfacer completamente las necesidades } \\
\text { básicas. } \\
\text { Exceso de control sobre los usuarios }{ }^{27} \text {. }\end{array}$ & $\begin{array}{l}\text { El paradigma que se utiliza es el Reactivo- curati- } \\
\text { vo, no se enfoca en la prevención }{ }^{15} \text {. }\end{array}$ \\
\hline Reglas $^{18,34}$ & $\begin{array}{l}\text { Obligan a los habitantes a estar la mayor parte } \\
\text { del tiempo del día en comportamientos seden- } \\
\text { tarios: } \\
\text { Periodos de descanso muy prolongados. Ac- } \\
\text { tividades recreativas con muy bajo gasto me- } \\
\text { tabólico. Uso de restricciones físicas y farma- } \\
\text { cológicas. }\end{array}$ & $\begin{array}{l}\text { Las actividades de entretenimiento pasivo, como } \\
\text { juegos de mesa, ver televisión y actividades reli- } \\
\text { giosas }^{34} \text {. }\end{array}$ \\
\hline $\begin{array}{l}\text { Desconocimiento de las prefe- } \\
\text { rencias de los residentes } \\
\end{array}$ & $\begin{array}{l}\text { No permite mejorar la prestación de servicios } \\
\text { de la institución. } \\
\text { No se utiliza la filosofía de Atención Centrada } \\
\text { en la Persona. }\end{array}$ & $\begin{array}{l}\text { Los centros de institucionalización que han imple- } \\
\text { mentado en modelo centrado en la persona son } \\
\text { preferidos por los usuarios que nos que no utilizan } \\
\text { esta filosofía } O R=2,47, p=0,005^{41} \text {. }\end{array}$ \\
\hline
\end{tabular}




\begin{tabular}{|c|c|c|}
\hline Aspecto & Características & Observaciones \\
\hline \multirow[t]{2}{*}{ Ausencia de programas ${ }^{17}$} & $\begin{array}{l}\text { Falta de estimulación cognitiva. Falta de even- } \\
\text { tos que estimulen la interacción familiar y so- } \\
\text { cial }^{18} \text {. Incapacidad para realizar actividades } \\
\text { recreativas y depor- tivas que estimulen la } \\
\text { actividad física }{ }^{18} \text {. }\end{array}$ & \multirow[t]{2}{*}{$\begin{array}{l}\text { Los programas de rehabilitación física mejoran e } \\
\text { rendimiento en el índice de Barthel ( } 0 \text { a } 100) \text { punta- } \\
\text { jes de seis puntos (intervalo de confianza del } 95 \% \\
2-11, p=0,008^{46} \text {. }\end{array}$} \\
\hline & $\begin{array}{l}\text { Falta de programas de rehabilitación para a } \\
\text { mantener o mejorar el desem- peño del usua- } \\
\text { rio en las } A B V D^{18,19,34,37} \text {. }\end{array}$ & \\
\hline $\begin{array}{l}\text { Personal de atención } \\
\text { directa }^{14,17,19,21,41,49}\end{array}$ & $\begin{array}{l}\text { Personal de asistencia con escasa especiali- } \\
\text { zación según grupo etario, falta de capacita- } \\
\text { ción }{ }^{17} \text {. }\end{array}$ & $\begin{array}{l}\text { Existe menor deterioro funcional cuando hay inter- } \\
\text { vención de un médico geriatra en la institución, OR } \\
0,60, \mathrm{Cl} 95 \% \quad 0,41-0,88, p=0,015^{49} \text {. }\end{array}$ \\
\hline Situación económica ${ }^{19,21,45}$ & $\begin{array}{l}\text { Las instituciones presentan una situación eco- } \\
\text { nómica usualmente limitada. La falta de re- } \\
\text { cursos económicos está relacionada con una } \\
\text { disminución en la calidad de servicio que se } \\
\text { brinda. }\end{array}$ & $\begin{array}{l}\text { En Brasil los hogares filantrópicos destinan un } 52 \% \\
\text { de sus recursos para el pago de salarios del perso- } \\
\text { nal lo que disminuye las posibilidades de invertir en } \\
\text { la calidad de los servicios }{ }^{45} \text {. }\end{array}$ \\
\hline Tipo de intervención ${ }^{16,19}$ & $\begin{array}{l}\text { Estándares de funcionamiento de la institu- } \\
\text { ción frente a una población heterogénea. }\end{array}$ & $\begin{array}{l}\text { No se realizan intervenciones individualizadas }(p= \\
-0,09)^{32} \text {. }\end{array}$ \\
\hline Horarios $^{27,30}$ & $\begin{array}{l}\text { Horarios fijos para la realización de las activi- } \\
\text { dades cotidianas. }\end{array}$ & $\begin{array}{l}\text { Solo el } 25 \% \text { de los residentes decide la hora de } \\
\text { ducharse }{ }^{27} \text {. }\end{array}$ \\
\hline Población ${ }^{17,19}$ & $\begin{array}{l}\text { La sobrepoblación, se deben satisfacer las ne- } \\
\text { cesidades de muchos en poco tiempo. }\end{array}$ & $\begin{array}{l}\text { En Korea por cada } 25 \text { residentes debe haber un } \\
\text { asistente de enfermería certificado }{ }^{19} \text {. }\end{array}$ \\
\hline
\end{tabular}

ABVD: actividades básicas de la vida diaria

Fuente: elaboración propia basada en referencias: 7, 9, 11, 14-16, 18, 21-29, 31-33, 36- 44, 46, 47, 49, 50.

Por último, pero no menos importante, están los factores ambientales que representan riesgo para los TM en PAMI. Baert y cols. ${ }^{32}$ y González y cols. $^{18}$, identifican algunos factores ambientales que podrían generar influencia en el desarrollo del DF de las PAMI, al mencionar aspectos ambientales se refieren directamente a la infraestructura o arquitectura de los centros de institucionalización.

Fonseca y cols. ${ }^{45}$ y Ausserhofer y cols. ${ }^{44}$ manifiestan que el diseño adecuado de los espacios físicos y la organización de los mismos proporcionan al residente la apreciación de sentirse como en casa, mejora la seguridad y proporciona mayor satisfacción respecto a los servicios recibidos.

En contraparte la infraestructura inadecuada ya sea por ausencia de espacios o bien porque los mismos presenten diseños inadecuados al no ser accesibles para todos, constituye una barrera para la ejecución de planes de actividad física o bien programas de rehabilitación física y funcional que proporcionen mayor calidad de vida a los habitantes lo que influye directamente en el desarrollo de patrones de movimiento inadecuados, entre ellos en la marcha ${ }^{18}$.

Baert y cols. ${ }^{32}$ en su estudio realizado en el 2016, evaluó las razones que manifestaban los organizadores de los centros de institucionalización para justificar la no realización de planes de actividad física para los residentes, entre otras la falta de jardines en un $53 \%$, ausencia de escaleras $44 \%$, espacio insuficiente $42 \%$ e infraestructura antigua en un $40 \%$ se manifestaron como deficiencias en los espacios físicos que se comportan como barreras para la realización de actividades que estimulen la movilidad, siendo esto último esencial para el mantenimiento o mejora del desempeño funcional de los institucionalizados. Lo mencionado anteriormente confirma la valoración de Preciado 
${ }^{38}$ quien asegura que en las residencias geriátricas prevalece el incumplimiento de los requerimientos arquitectónicos para todos.

El enlace de las características intrínsecas de las PAMI con los aspectos de la dinámica de los sistemas de institucionalización, desencadenan alteraciones en los patrones de movimiento de los residentes. En la figura 3 se detalla la relación entre los aspectos antes mencionados y su influencia en el patrón de marcha de los usuarios.

\section{Discusión}

Un AM institucionalizado se caracteriza en gran medida por DF, discapacidad física, dependencia de moderada a severa en las actividades básicas de la vida diaria y la elevada incidencia de comorbilidad. El sistema de institucionalización aporta un paradigma de atención en el cual no se estimula la realización de programas de rehabilitación dirigidas a mejorar la capacidad física del individuo, por el contrario se propicia el sedentarismo. Asimismo, el usuario es un receptor pasivo de los servicios. Como consecuencia el deterioro físico se acelera y su manifestación más evidente se desencadena en el patrón de marcha con una disminución en la velocidad y longitud de paso, pérdida de equilibrio, cambios posturales, seudoclaudicación y asimetría en el movimiento ${ }^{6-9,11,14-20,22-28,30-34,36,38,39,41-44,46 . ~}$

La preservación de la marcha es uno de los requisitos más importantes para valorar el estado de salud y la calidad de vida de un AM, el inicio de un trastorno podría referenciarse ante una marcha lenta, inestable o comprometida desde el punto de vista biomecánico, lo suficientemente ineficaz para un desplazamiento con normalidad.

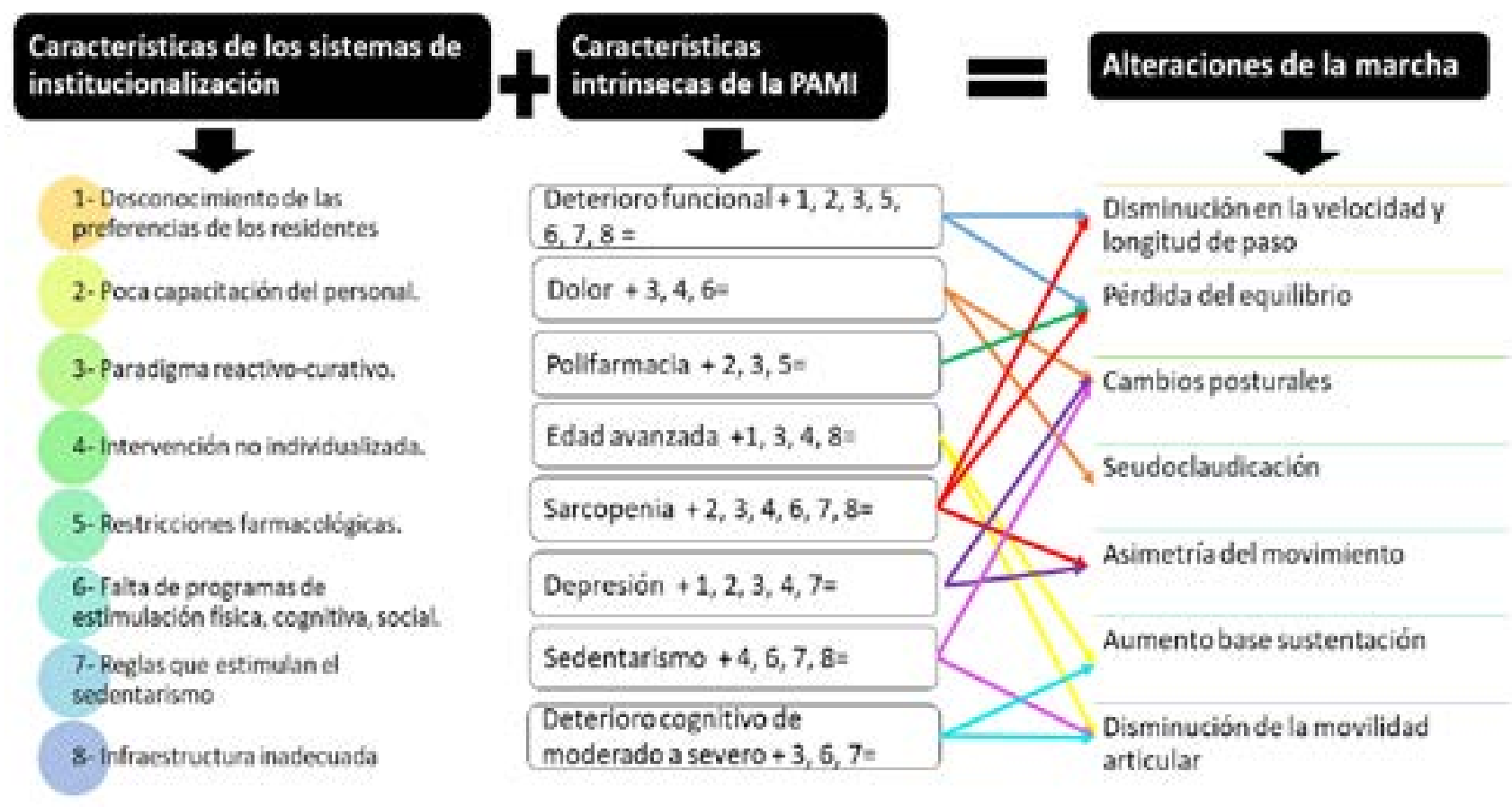

Figura 3: Relación entre las características de la persona adulta mayor institucionalizada y la influencia del sistema de institucionalización con las alteraciones de la marcha.

Fuente: elaboración propia basada en referencias 5- 7, 9, 10, 11, 14-18, 20-29, 31- 34, 36- 44, 46-51. 
La edad de los residentes suele ser, en promedio, mayor a los 80 años y se ha determinado que la prevalencia de los TM aumenta con la edad en un $50 \%$. A esto se le suma que, el personal de atención de los centros de institucionalización suelen mantener una carente especialización según la población a la cual se dirigen, por lo que la posible intervención de las alteraciones podría ser deficiente e inoportuna ${ }^{5,7-10,14-18,21,23-25,28,29,31,32,38,40,43}$.

La falta de estimulación cognitiva, la disminución de la interacción social y familiar, la falta de intervenciones individualizadas; y las actividades rutinarias fijas sin cambios en su proceder, se unen al deterioro cognitivo moderado - severo de la población institucionalizada que sin duda influyen en la marcha al propiciar una dificultad para iniciar la misma, la desviación de la trayectoria, pérdida de equilibrio, entre otras ${ }^{6,7,11,14-19,23,26,27,30,31,34,36-39,43,44,45}$.

La infraestructura insuficiente e inadecuada según las necesidades de los residentes aumenta el riesgo de caída en los $\mathrm{AM}$ que según la evidencia presenta una prevalencia de caídas de un 30 a $50 \%$ de la población institucionalizada, dicho riesgo incrementa con la deficiencia de programas que estimulen la actividad física ${ }^{15,16,18-20,22,23,29,30,32,38,44,45,48}$.

Kim y cols. ${ }^{20}$ determinan una relación directa entre el DF, la depresión y la calidad de vida de los AM institucionalizados, sobre todo cuando el personal de atención no valida ni conoce las preferencias de los residentes. De igual manera, ante el aislamiento por la falta de interacción social y familiar, un AM con sintomatología depresiva presenta un peor desempeño físico y funcional, lo que afecta su deambulación ${ }^{6,11,14-16,18,20-22,25,27-29,34,36,39-41,43-45,47 . ~}$

El patrón de marcha también se afecta ante la presencia de sarcopenia y fragilidad, grandes síndromes geriátricos presentes en la población institucionalizada, para los cuales el sedentarismo, la falta de estimulación física, cognitiva y social así como la dinámica institucional desencadenan mayor deterioro $6,8,11,14-16,18,21,22,27-29,31-34,36-40,43,44$.

Lautilización de restriccionesfísicas y farmacológicas generan actitudes sedentarias en los usuarios, Laffón y cols. ${ }^{13}$ promueven un modelo enfocado en la prevención de la inactividad física como un factor protector del deterioro físico, funcional y cognitivo, así como intervenciones individualizadas y ejecutadas por personal de atención con perfil académico especializado en la población meta; todo como parte de un replanteamiento del paradigma de intervención de los centros de residencia para PAM $^{7,9,15,16,18,19,21-24,28,31,32,34,38-40,43,44,45 . ~}$

El 53\% de la población AM institucionalizada indica sentir dolor, el mismo desencadena un patrón de marcha típico de seudoclaudicación, puede haber asimetría del movimiento, disminución de la velocidad, así como resistencia a la ejecución de la marcha, por lo que una inadecuada intervención de las manifestaciones dolorosas influye directamente en el desempeño funcional así como en la calidad de vida del AM. Por lo tanto, una intervención individual y especializada será la mejor opción de tratamiento para dichas alteraciones ${ }^{6,7,11,15,16,19-24,28,32,38-40,44,45}$.

Esta revisión básicamente destaca la existencia de factores de riesgo para los TM en PAMI tales como las características intrínsecas de las personas residentes de los centros de institucionalización, aspectos propios de la dinámica de los hogares de larga estancia para población geriátrica y por último factores de infraestructura que generan un deterioro físico y funcional que influye directamente en la correcta realización de patrones de movimiento tales como la marcha.

Ante el acelerado crecimiento de la población adulta mayor en general y por ende la institucionalizada, es fundamental que las autoridades competentes generen políticas estatales e institucionales que promuevan modelos de atención destinados a 
promover la autonomía del AM, la actividad física, estimulación cognitiva, social y familiar para que los usuarios de los centros de atención posean un mejor desempeño funcional y por ende una mejor calidad de vida, reflejada en la ejecución de patrones motores como la marcha.

\section{Referencias bibliográficas}

1. Velasco R, Bejines M, Sánchez R, Mora A, Benítez V, García L. Envejecimiento y capacidad funcional en adultos mayores institucionalizados del occidente de México. Nure Inv [Internet]. 2015 [citado 1 abril 2017];12(74):1-11. Disponible en: http://www.nureinvestigacion.es/OJS/index.php/ nure/article/view/86

2. Valera SG. Instrumento de valoración gerontológica para el proceso de institucionalización en el hogar Carlos María Ulloa: país: Costa Rica. Humanit Rev Investig [Internet]. 2014 [citado 17 abril 2017];11(11):99-116. Disponible en: https://dialnet.unirioja.es/servlet/articulo?codigo $=4866935$

3. Instituto Nacional de Estadísticas y Censos (CR). INEC, estimaciones y proyecciones de Costa Rica por sexo y edad 1950 - 2050 [Internet]. San José, Costa Rica: Instituto Nacional de Estadísticas y Censos; 2017. Disponible: http://www.inec. go.cr/sites/default/files/infografias-pdf/imgadultomayor2809207_0.pdf

4. Opinión de experto: Dra. Cleidy Monge Cantillo, MSC enfermera unidad servicios de salud, atención directa a las personas. Dirección de garantía de acceso a los servicios de salud. Ministerio de Salud Costa Rica. 18 abril 2018.

5. Calero PA, Chaves MA. Cambios fisiológicos de la aptitud física en el envejecimiento. Revista Investig Salud Univ Boyacá [Internet]. 2016 [citado 10 de junio de 2018];3(2):176-194. Disponible en: http://revistasdigitales.uniboyaca.edu.co/index.php/rs/article/view/178
6. Cerda L. Manejo del trastorno de la marcha del adulto mayor. Rev Med Clin Condes [Internet]. 2014 [citado 31 de mayo de 2018];25(2):265-275. Disponible en https://ac.elscdn.com/ S07168640 14700379/1-s2.0-S0716864014700379-main. pdf?_tid=e1671afa-960c-4935-937c-b99c8ced25ba\&acdnat $=1527767110$ _ feca9219cfa9886 ceb80fdc7d46a155d.

7. Agudelo A, Briñez T, Guarín V, Ruiz J, Zapata M. Marcha: descripción, métodos, herramientas de evaluación y parámetros de normalidad reportados en la literatura. CES Mov Salud [Internet]. 2013 [citado 19 de septiembre];1(1):29-43. Disponible en: http://revistas.ces.edu.co/index.php/ movimientoysalud/article/view/2481

8. Buckinx F, Croisier J, Reginster J, Lenaerts C, Brunois $T$, Rygaert $X$ et al. Prediction of the incidence of falls and deaths among elderly nursing home residents: The SENIOR study. J Am Med Dir Assoc [Internet]. 2018 [citado 22 mayo 2018];19(1):18-24. Disponible en: https://www. ncbi.nlm.nih.gov/pubmed/28757332

9. Kuys S, Peel N, Klein K, Slater A, Hubbard R. Gait speed in ambulant older people in long term care: a systematic review and meta-analysis. J Am Med Dir Assoc [Internet]. 2014 [citado 19 junio 2018];15(3):194-200. Disponible en: https://www.sciencedirect.com/science/article/pii/ S1525861013006075

10. Mailyn J. Calderón D, Ricardo A. Ulloa J. Cambios asociados al envejecimiento normal en los parámetros angulares de la marcha a una velocidad controlada. Rev Med Chile [Internet]. 2016 [citado octubre de 2015];144:7482. Disponible en: https://scielo.conicyt.cl/ scielo.php?script=sci_abstract $\&$ pid $=$ S0034$98872016000100010 \&$ Ing=es\&nrm=iso\&tlng=es

11. Enríquez M, Cruz J, Celestino Soto, Celestino M, Garza M, Salazar M. Función Ejecutiva, Velocidad de la Marcha y Tarea Doble en Adultos Mayores Mexicanos. Rev Iberoam Psicol Ejerc Deporte [Internet]. 2013 [citado 15 de junio de 
2018];8(2):345-357. Disponible en: http://www. redalyc.org/articulo.oa?id=311128824006

12. Medina P, Muñoz R, Escobar M. Reserva funcional en adultos mayores autovalentes: una estimación de la velocidad y el costo fisiológico de marcha. Rev Bras Geriatr Gerontol [Internet]. 2016 [citado 20 de junio de 2018];19(4):577-589. Disponible en: https://www.researchgate.net/profile/ Paul_Medina_Gonzalez/publication/308356260_ Reserva_funcional_en_adultos_mayores_autovalentes_una_estimacion_de_la_velocidad_y_el_costo_fisiologico_de_marchal links/57e1c54508ae1f0b4d93f3fc/Reserva-uncional-en-adultos-mayores-autovalentes-una-estimacion-de-la-velocidad-y-el-costo-fisiologico-de-marcha.pdf

13. Rodríguez G, Burga D, Cipriano G, Ortiz P, Tello $T$, Casas $P$ et al. Factores Asociados a Velocidad de Marcha Lenta en Adultos Mayores en un Distrito de Lima, Perú. Rev Peru Med Exp SaIud Pública [Internet]. 2017 [citado 20 de junio de 2018];34(4):619-626. Disponible en : http://www. rpmesp.ins.gob.pe/index.php/rpmesp /article/ view /30 $25 / 2880$

14. Naylor M, Hirschman K, Hanlon A, Abbott K, Bowles K, Foust J. Factors associated with changes in perceived quality of life among elderly recipients of long-term services and supports. J Am Med Dir Assoc [Internet]. 2015 [citado 24 mayo 2018];17(1):44-52. Disponible en: https:// www.jamda.com/article/S1525-8610(15)004983/fulltext

15. Laffon C, Morley J, Levy C, Agenes F, Barbagallo $\mathrm{M}$, Cerati $\mathrm{M}$ et al. Prevention of functional decline by reframing the role of nursing homes?. J Am Med Dir Assoc [Internet]. 2017 [citado 24 mayo 2018];18(2):105-110. Disponible en: https://www. jamda.com/article/S1525-8610(16)30551-5/fulltext

16. Fedecostante M, Dell'aquila G, Eusebi P, Volpato S, Zuliani G, Abete P et al. Predictors of functional changes in Italian nursing home residents: the
U.L.I.S.S.E. study. J Am Med Dir Assoc [Internet]. 2015 [citado 11 junio 2018];17(4):306-311. Disponible en: https://www.jamda.com/article/ S1525-8610(15)00704-5/fulltext

17. Palase A, Menegazzi G, Tullio A, Zigotti M, Hayter $M$, Watson R. Functional decline in residents living in nursing homes: a systematic review of the literature. J Am Med Dir Assoc [Internet]. 2016 [citado 24 mayo 2018];17(8):694-705. Disponible en: https://www.jamda.com/article/ S1525-8610(16)30087-1/fulltext

18. González M, Meillon C, Rullier L, Ávila J, Bergua $\mathrm{V}$, Dartigues $\mathrm{J}$ et al. Cognitive decline after entering a nursing home: a 22 year follow-up study of institutionalized and noninstitutionalized elderly people. J Am Med Dir Assoc [Internet]. 2014 [citado 24 mayo 2018]:15(7):504-508. Disponible en: $\quad$ https://www.jamda.com/article/S15258610(14)00117-0/fulltext

19. Lee T, Cho E, Shil E, Sun H, Kyung Y, Nam B et al. Activities of daily living in nursing home and home care settings: a retrospective 1-year cohort study. J Am Med Dir Assoc [Internet]. 2014 [citado 24 mayo 2018];16(2):114-119. Disponible en: https:// www.jamda.com/article/S1525-8610(14)00472$1 /$ fulltext

20. Kim S, Park E, Kim S, Nakagawa S, Lung J, Bum $\mathrm{J}$ et al. The association between quality of care and quality of life in long-stay nursing home residents with preserved cognition. J Am Med Dir Assoc. [Internet]. 2014 [citado 25 mayo 2018];15(3):220-225. Disponible en: https://www. jamda.com/article/S1525-8610(13)00595-1/pdf

21. Tanuseputro P, Hsu A, Kuluski K, Chalifoux M, Donskov M, Beach $S$ et al. Level of need, divertibility, and outcomes of newly admitted nursing home residents. J AM Med Dir Assoc [Internet]. 2017 [citado 24 mayo 2018];18(7):616-623. Disponible en: https://www.jamda.com/article/ S1525-8610(17)30103-2/fulltext

22. De Souto P, Morley J, Chodzko W, Pitkala K, 
Weening E, Rodríguez $L$ et al. Recommendations on Physical Activity and Exercise for Older Adults Living in Long-Term Care Facilities: A Taskforce Report. J Am Med Dir Assoc [Internet]. 2016 [citado 29 Mar 2017];17(5):381-92. Disponible en: http://www.jamda.com/article/S15258610(16)00059-1/abstract

23. Holup A, Hyer K, Meng H, Volicer L. Profile of nursing home residents admitted directly from home. J Am Med Dir Assoc [Internet]. 2017 [citado 23 mayo 2018];18(2):131-137. Disponible en: $\quad$ https://www.jamda.com/article/S15258610(16)30369-3/abstract

24. Matus M, Cid C. Building long term care policies in Latin America: New programs in Chile. J Am Med Dir Assoc [Internet]. 2015 [citado 11 junio 2018];16(10):900.e7-900.e10. Disponible en: $\quad$ https://www.jamda.com/article/S15258610(15)00497-1/fulltext

25. Martin R, Hayes B, Gregorevic K, Kwang W. The effects of advance care planning interventions on nursing home residents: a systematic review. J AM Med Dir Assoc [Internet]. 2016 [citado 24 mayo 2018];17(4):284-293. Disponible en: https:// www.jamda.com/article/S1525-8610(15)007768/fulltext

26. Santos B, Meylan L. Older citizens opinions on long-term care options: vignette survey. J Am Med Dir Assoc [Internet]. 2017 [citado 22 mayo 2018];18(4):326-334. Disponible en: https://www. ncbi.nlm.nih.gov/pubmed/27956072

27. Morris J, Declercq A, Hirdes J, Finne H, Fries $B$, James $M$ et al. Hearing the voice of the resident in long term-care facilities- an internationally based approach to assessing quality of life. J Am Med Dir Assoc [Internet]. 2017 [citado 24 mayo 2018];19(3):207-215. Disponible en: https://www. jamda.com/article/S1525-8610(17)30464-4/fulltext

28. McGilton $\mathrm{K}$, Bowers $\mathrm{B}$, Heath $\mathrm{H}$, Shannon $\mathrm{K}$, Dellefield M, Prentice D et al. Recommendations from the international consortium on professional nursing practice in long-term care homes. J Am Med Dir Assoc [Internet]. 2015 [citado 11 junio 2018];17(2):99-103. Disponible en: https://www. jamda.com/article/S1525-8610(15)00701-X/fulltext

29. São L, Nogueiro A, Mendes M, Pinto A, Pimentel $M$. Deterioro funcional, miedo a caerse y composición corporal en ancianos institucionalizados. Enferm Clin [Internet]. 2015 [citado 17 abril 2017];25(2):81-6 Disponible en: http://www. elsevier.es/es-revista-enfermeria-clinica-35-articulo-deterioro-funcional-miedo-caerse-composicion-S1130862114001442

30. Souza J, Mahecha S, Leandro T. El efecto de la inactividad física en la aptitud física y funcional en personas mayores institucionalizados de São Caetano do Sul, São Paulo, Brasil. Rev Cienc Activ Física [Internet]. 2014 [citado 14 abril 2018];15(2):63-72. Disponible en: https://www.researchgate.net/ publication/283655484_The_effect_of_physical_activity_on_the_physical_and_functional_fitness_in_institutionalized_adults_greater_of_ Sao_Caetano_do_Sul_Sao_Paulo_Brazil

31. Cherubini A, Corsonello A, Lattanzio F. Polypharmacy in Nursing home residents: what is the way forward? J Am Med Dir Assoc [Internet]. 2016 [citado 11 junio 2018];17(11):4-6. Disponible en: https://www.jamda.com/article/S15258610(15)00477-6/fulltext

32. Baert V, Gorus E, Calleeuw K, De Backer W, Bautmans I. An administrators perspective on the organization of physical activity for older adults in long-term care facilities. J Am Med Dir Assoc [Internet]. 2016 [citado 1 abril 2017];17(1):7584. Disponible en: https://www.ncbi.nlm.nih.gov/ pubmed/26432625

33. Chang K, Tsai-Hsuan H, Wei-Ting W, Kuo-Chin H, Der-Sheng $\mathrm{H}$. Association Between Sarcopenia and Cognitive Impairment: A Systematic Review and Meta-Analysis. J Am Med Dir Assoc [Internet]. 
2016 [citado diciembre de 2016];17(12):1164. e7-1164.e15. Disponible en: https://www.jamda. com/article/S1525-8610(16)30428-5/fulltext

34. Furtado H, Sousa N, Simão R, Dutra F, VilaçaAlves J. Physical exercise and functional fitness in independently living vs institutionalized elderly women: a comparison of 60- to 79-year-old city dwellers. Clin Interv Aging [Internet]. 2015 [citado 24 abril 2015];10:795-801. Disponible en: https://www.dovepress.com/physical-exercise-and-functional-fitness-innbspindependently-living-vs-peer-reviewed-article-CIA

35. Pesantes Rodríguez V. Guía de ejercicios terapéuticos para reeducar la marcha en pacientes de la tercera edad en la ciudad de Guayaquil [Tesis de Maestría en Educación Superior e Investigación Educativa]. Guayaquil, EC: Universidad de Guayaquil; 2016. Disponible en: http://repositorio.ug.edu.ec/bitstream/redug/27068/1/EC-UGPOS-DP-MDUIE-003.pdf

36. Quintar E, Giber F, Las caídas en el adulto mayor: factores de riesgo y consecuencias. Actual Osteol [Internet]. 2014 [citado enero 2014];10(3):278286. Disponible en: http://www.sanatorioallende.com/web/ES/las_caidas_en_adultos_mayores_y_sus_consecuencias.aspx

37. Sitjà $M$, Martínez $M$, Fort $A$, Rey $F$, Romero $D$, Bonfill X. Effects of a Whole Body Vibration (WBV) Exercise Intervention for Institutionalized Older People: A Randomized, Multicentre, Parallel, Clinical Trial. J Am Med Dir Assoc [Internet]. 2015 [citado 29 Mar 2017];16(2):125-31. Disponible en: http://www.jamda.com/article/S15258610(14)00477-0/pdf

38. Preciado S. El adulto mayor, las casas de atención y la propuesta de un modelo de atención social: revisión de su aplicación y propuestas. Rumbo TS [Internet]. 2014 [citado 25 abril 2018];9:7997. Disponible en: https://www.researchgate.net/ publication/275334347_El_adulto_mayor_las_ casas_de_atencion_y_la_propuesta_de_un_ modelo_de_atencion_social_revision_de_su_ aplicacion_y_propuestas

39. Guevara N. Impactos de la institucionalización en la vejez. Análisis frente a los cambios demográficos actuales. Entramado [Internet]. 2016;12(1):138-151. Disponible en: http://www. redalyc.org/articulo.oa?id=265447025009

40. Sánchez García MR. Fin de vida en residencias de ancianos desde la perspectiva de los profesionales: dificultades, factores favorables y calidad de los cuidados al final de la vida [Tesis de Doctorado]. Granada, España: Universidad de Granada; 2017. Disponible en: http://digibug.ugr. es/bitstream/handle/10481/47713/2675678x.pdf?sequence $=1$ \&isAllowed $=y$

41. Poey J, Hermer L, Cornelison L, Kaup M, Drake $\mathrm{P}$, Stone $\mathrm{R}$ et al. Does person centered care improve residents satisfaction with nursing home quality? J Am Med Dir Assoc [Internet]. 2017 [citado 24 mayo 2018];18(11):974-979. Disponible en: $\quad$ https://www.jamda.com/article/S15258610(17)30338-9/fulltext

42. Díaz Unzueta E. Modelo gerontológico de atención al adulto mayor desde el enfoque centrado en la persona. [Tesis de Maestría en Desarrollo Humano]. Jalisco: Instituto Tecnológico y de Estudios Superiores de Occidente; 2016. Disponible en: https://rei.iteso.mx/bitstream/handle/11117/4033/MDH55509. pdf?sequence $=2$

43. Beauchet $O$, Annweiler $C$, Callisaya M, De Cock A, Helbostad J, Kressig R et al. Poor gait performance and prediction of dementia: results from a meta-analysis. J Am Med Dir Assoc [Internet]. 2016 [citado 11 junio 2018];17(6):482-490. Disponible en: https://www.jamda.com/article/ S1525-8610(15)00851-8/abstract

44. Ausserhofer D, Deschodt M, De Geest S, Van Achterberg T, Meyer G, Verbeek H et al. "There's No Place Like Home": A Scoping Review on the Impact of Homelike Residential Care Models on Resident-, Family-, and Staff-Related Outcomes. J Am Med Dir Assoc [Internet]. 2016 [citado 
1 de agosto 2016];17(8):685-693. Disponible

en: $\quad$ https://www.jamda.com/article/S1525-

8610(16)30048-2/fulltext

45. Fonseca W, Saturno P, de Meneses K, da Silva G, da Silva Z. Quality of care in nursing homes in Brazil. J Am Med Dir Assoc [Internet]. 2017 [citado 24 mayo 2018];18(7):636e13-636e19. Disponible en: https://www.jamda.com/article/ S1525-8610(17)30222-0/fulltext

46. CrockerT, Foster A, Youg J, Brown L, Ozer S, Smith $\mathrm{J}$ et al. Physical rehabilitation for older people in long-term care. Cochrane Database Syst Rev [Internet]. 2013 [citado 28 de febrero 2013]. Disponible en: http://eprints.whiterose. ac.uk/75591/2/CD004294.pdf

47. Sheppard Ch, McArthur C, Hitzing S. A systematic review of Montessori-Based activities for persons with dementia. J Am Med Dir Assoc [Internet]. 2015 [citado 24 mayo 2018];17(2):117-122. Disponible en: https://www.jamda.com/article/ S1525-8610(15)00643-X/abstract

48. Monge T, Solís Y. El síndrome de caídas en persona adultas mayores y su relación con la velocidad de la marcha. Rev Med Costa Rica Centroam [Internet]. 2016 [citado 20 de junio del 2018];73(618):91-95. Disponible en: http://www. medigraphic.com/pdfs/revmedcoscen/rmc-2016/ rmc161r.pdf

49. Giai M. Actividades recreativas en adultos mayores internos en un hogar de ancianos. Rev Cub Salud Pública [Internet]. 2015 [citado 24 mayo 2018];41(1):67-76. Disponible en: http://scielo. sld.cu/scielo.php?pid=S0864-346620150001000 07\&script=sci_arttext\&tlng=pt

50. Guerrero M, Galván G, Vásquez F, Lázaro G, Morales $D$. Relación entre autoestima y autonomía funcional en función del apoyo social en adultos institucionalizados de la tercera edad. Psicogente [Internet]. 2015 [citado 25 abril 2018];18(34):303310. Disponible en: http://revistas.unisimon.edu. co/index.php/psicogente/article/view/1403
51. Zuliani M. Valoración geriátrica integral de los trastornos de la marcha y actividades de la vida diaria en adultos mayores. [Tesis de $\mathrm{Li}$ cenciatura en Kinesiología]. Buenos Aires: Universidad de Fasta; 2016. Disponible en: http://redi.ufasta.edu.ar:8080/xmlui/bitstream/ handle/123456789/1302/2016_K_054.pdf?sequence $=1$ 